\title{
Caging dynamics in bacterial colonies
}

\author{
Marc Hennes $\odot$, ${ }^{*}$ Tom Cronenberg, and Berenike Maier $₫$ \\ Institute for Biological Physics, and Center for Molecular Medicine Cologne, University of Cologne, Cologne, Germany
}

(Received 3 September 2021; accepted 21 January 2022; published 8 March 2022)

\begin{abstract}
In colonies, bacteria are often densely packed due to strong attractive forces between neighboring cells. For organisms where aggregation is mediated by type 4 pili interactions, the pilus-mediated motor activity fluidizes these colonies, but it is unclear how close packaging affects bacterial motility. Here, we characterize the dynamics of single cells over five orders of magnitude in time. At short timescales, we find pilus-driven ballistic dynamics, which depending on the volume fraction of cells, transitions to caged or diffusive dynamics at intermediate timescales. Long-term dynamics are dominated by growth-induced radial motion overcoming caging at high packings. Reducing attractive interactions by antibiotic treatment delays caging and introduces long-ranged collective motion.
\end{abstract}

DOI: 10.1103/PhysRevResearch.4.013187

\section{INTRODUCTION}

Bacterial colonies share similarities with densely packed colloidal systems, in which the structure, dynamics, and phase behavior are governed by the volume fraction of particles. At lower fractions, the systems behave like fluids. Beyond a critical density, particles become topologically trapped within cages formed by surrounding colloids [1]. Particles move freely inside the interparticle solvent space before the system experiences a dynamic arrest commonly referred to as caging. This dynamic slowing down becomes transient as neighbor structures relax, and particles diffuse at longer timescales [1]. Similar evolutions are encountered across a wide range of systems at higher packings, e.g., attractive [1], granular [2], or driven systems. For dense self-propelled colloids, the local energy injection influences the onset of caging at similar volume fractions [3]. In this paper, we investigate a system combining driven colloidal motion and attractive interactions.

The human pathogens Neisseria gonorrhoeae and Neisseria meningitidis are spherically- or dumbbell-shaped bacteria which form densely packed three-dimensional (3D) colonies [4]. Within these colonies, cells exert localized attractive interactions by means of type 4 pili (T4P), extracellular polymers that generate high forces by retraction $[5,6]$. T4P of neighboring cells continuously bind, retract, and detach [7]. This leads to driven and stochastic cell trajectories. In contrast to other active matter systems [8-10], cell motility does not result from monopole propulsion forces but from a tug-of war mechanism between multiple pilus connections, captured

\footnotetext{
*marc.hennes@uni-koeln.de

Published by the American Physical Society under the terms of the Creative Commons Attribution 4.0 International license. Further distribution of this work must maintain attribution to the author(s) and the published article's title, journal citation, and DOI.
}

as a sum of pairwise fluctuating attractive interactions [11]. This tug-of-war enables liquidlike behavior of colonies at long timescales [12]; aggregated cells exhibit local structural order, and nearby colonies coalesce with liquidlike relaxation dynamics [13-15]. Previous efforts have focused on recreating these material properties and fusion dynamics numerically, using single-cell [14,15] and coarse-grained simulations [16]. Both experiments and simulations have addressed motility of single bacteria within gonococcal and meningococcal colonies. At a timescale of single T4P retraction, i.e., 1-10 $\mathrm{s}$, these single-cell trajectories were described as diffusive, whereby the diffusivity is heterogeneous within the colony $[14,15,17]$. However, it is currently unknown whether the trajectories are diffusive beyond this limited timescale. The tug-of-war between cells may not simply be mappable to an effective equilibrium attractive potential, and correlation times and lengths of persistent retraction events within the colony are unknown. On intermediate timescales, it is not clear how diffusive dynamics arise within colonies of trapped cells. Caging is likely to affect bacterial physiology because it interferes with cellular proliferation in agreement with previously observed growth arrest [18]. Therefore, it will be important to study bacterial dynamics beyond the timescale of a single T4P retraction event.

In this paper, we characterize experimental trajectories inside bacterial clusters for times ranging from $10^{-2}$ to $10^{3}$ $\mathrm{s}$ using velocity correlation functions and the mean-squared displacement (MSD) of cells. We show that pilus retractions induce a short-term persistent regime followed by backscattering. At high volume fractions, we find a transition to a caged regime at intermediate timescales. The caging regime is transient; at long timescales, the continuous caged dynamics is overcome by the radial expansion velocity field mediated by cell division. At low volume fractions, we observe a transition to a diffusive regime in which the diffusion constant scales with the inverse squared volume fraction. The antibiotic azithromycin reduces attractive interactions between neighboring cells [17]. We show that azithromycin shifts the 
TABLE I. Bacterial strains used in this paper.

\begin{tabular}{|c|c|c|}
\hline Strain & Relevant genotype & Source/reference \\
\hline$w t^{*}(\mathrm{Ng} 150)$ & G4::aac & [33] \\
\hline$w t *$ green $(\mathrm{Ng} 194)$ & $\begin{array}{c}\text { lctp: } P_{\text {pile }} \text { sfgfp speR:aspC } \\
\text { G4::aac }\end{array}$ & [18] \\
\hline$w t^{*}$ green $\Delta$ pilT $(\mathrm{Ng} 231)$ & $\begin{array}{c}\text { pilT::m-Tn3cm } \\
\text { lctp:P } P_{\text {pile }} \text { sfgfp speR:aspC } \\
\text { G4::aac }\end{array}$ & This paper \\
\hline
\end{tabular}

caging transition to later times and introduces long-ranged collective motion within colonies. We conclude that steric repulsion, pilus motor activity, and cell growth act together to induce transitions between dynamic regimes at different timescales, which are distinct from nonliving colloidal systems.

\section{BACTERIAL DYNAMICS WITHIN SPHERICAL COLONIES AT TIMESCALES FROM TENS OF MILLISECONDS TO HOURS}

We first investigated cell trajectories within colonies for times between 0.01 and $5 \mathrm{~s}$. To this end, $N$. gonorrhoeae cells (strain $\mathrm{Ng} 194$, see Table I) were incubated in a flow chamber under nutrient supply for $1 \mathrm{~h}$, which allowed clusters to settle in a spherical shape (materials and methods in Appendix). Cell trajectories were calculated from spinning disc confocal microscope image series [Fig. 1(a)] of the equatorial aggregate plane. In all cases, rigid-body colony translations and rotations were removed as described in Appendix. We studied correlations of the velocity of cells as a function of the lag time using the velocity autocorrelation function (VACF):

$$
C_{v}(\tau)=\left\langle\mathbf{v}_{i}(t+\tau) \cdot \mathbf{v}_{i}(t)\right\rangle_{t, i},
$$

with $\mathbf{v}_{i}(t)=\frac{\mathbf{r}_{i}(t+\Delta t)-\mathbf{r}_{i}(t)}{\Delta t}$. Averages were taken over times $t$ and cells $i$ in the bulk of the colony, defined as being at least $5 \mu \mathrm{m}$ away from the edge, where cell densities (Fig. 2) and previously determined diffusivities [17] are constant. The behavior at the periphery of the colony will be investigated in the next paragraph. For $\tau \lesssim 0.1 \mathrm{~s}$, we observe a positive plateau in $C_{v}(\tau)$ [Fig. 1(b)], which is absent for a strain lacking the retraction ATPase PilT (strain Ng231, see Table I). The persistent initial regime thus captures the pilus retraction activity. To extract characteristic decay times for $0 \leqslant \tau \leqslant 0.1 \mathrm{~s}$, we fitted the VACF to an exponential function $C_{v}(\tau) \sim \exp (-\tau / \tau c)$ and obtained a $\tau_{c}$ of $0.08 \pm 0.03 \mathrm{~s}\left(R^{2}=0.75\right)$, much shorter than for the motion of single surface-associated cells [11]. Beyond $\tau=0.1 \mathrm{~s}$, the VACF oscillates between positive and negative values, indicative of backscattering and caged dynamics. For $\tau \lesssim 1 \mathrm{~s}$, we characterized motility regimes using the MSD:

$$
\operatorname{MSD}(\tau)=2 \int_{0}^{\tau}(\tau-t) C_{v}(t) d t \sim \tau^{\alpha(\tau)},
$$

and its exponent $\alpha(\tau) \approx \frac{d \ln [\operatorname{MSD}(\tau)]}{d \ln (\tau)}$ in the adiabatic limit [Fig. 1(c)]. Calculating the MSD as an integral over the VACF simplifies the treatment of the tracking error, see Appendix. In line with the global shape of the VACF, $\alpha$ is superdiffusive $(\alpha>1)$ at short timescales and subdiffusive $(\alpha<1)$ for times $\tau>0.2 \mathrm{~s}>\tau_{c}$, again indicative of a transition from ballistic motion to caging.

This regime transition is reminiscent of the behavior of simple Newtonian liquids at low temperatures. An interesting difference in our active system is the fact that superdiffusive motion between collisions is created by T4P retractions. We hypothesized that caging is the result of steric contact interactions between cells [19]. We expect pili connections (a)

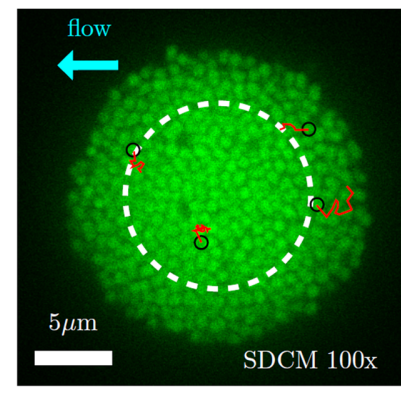

(c)

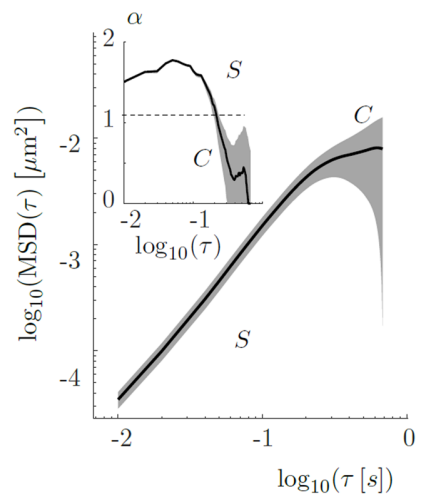

(b)

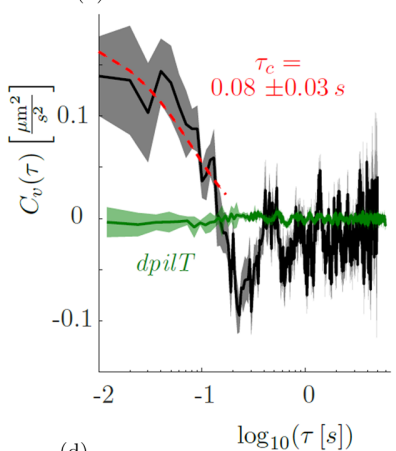

(d)

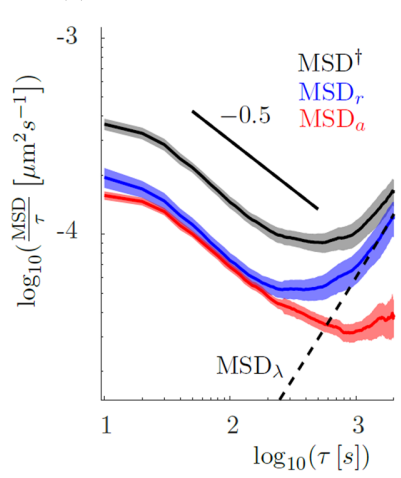

FIG. 1. Cell motility in the bulk of aggregates. (a) Fluorescence image of aggregated $N$. gonorrhoeae cells. Black circles: single bacteria, red lines: cell trajectories. The white dotted circle delimits the bulk. (b) $C_{v}(\tau)$ for $\tau$ between 0.01 and 5 s. Black: strain $(\mathrm{Ng} 194)$. Green: T4P retraction-deficient strain $(\mathrm{Ng} 231)$. Red dotted line: Exponential fit of the initial decay. (c) Mean-squared displacement (MSD) of bulk cells. Inlet: Regime exponent $\alpha(\tau) . S$ and $C$ indicate superdiffusive and caged regimes, respectively. (d) Total (black), radial (blue), and azimuthal (red) part of $\frac{\operatorname{MSD}(\tau)}{\tau}$ for times between 10 and 2000 s. Full black line: Caging with exponent $\alpha \approx 0.5$. Dotted black line: Growth contribution $\operatorname{MSD}_{\lambda}(\tau)$. Shaded regions indicate standard errors. 


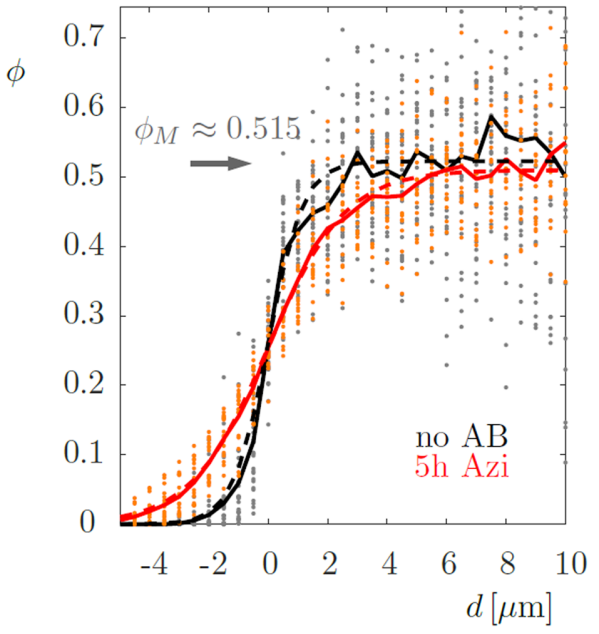

FIG. 2. Volume fraction $\phi$ as a function of distance from the edge of the colony $d$ for untreated (strain $\mathrm{Ng} 194$, no $\mathrm{AB}$, black points) and treated cells (strain $\mathrm{Ng} 150,5 \mathrm{~h}$ treatment with azithromycin at MIC $\times 100,6.4 \mu \mathrm{g} / \mathrm{mL}$, red points). Full lines: Averages over 30 colonies. Dotted lines: Fits to instantons.

to break for cells in close contact because the steric repulsion acts as a load force [13]. We calculated the cage radius $r_{\text {cage }}=\left[\frac{3}{2} \operatorname{MSD}\left(\tau_{\text {cage }}\right)\right]^{1 / 2}$, where $\tau_{\text {cage }}$ is the time at which $C_{v}$ becomes negative after the persistent plateau. We find values of $r_{\text {cage }} \approx 0.1 \mu \mathrm{m}$, which leads to next neighbor distances $r_{i j}=2 a_{\text {cell }}+2 r_{\text {cage }} \approx 1.2 \mu \mathrm{m}$, in line with the first maximum of the radial distribution function [17].

We then asked whether caging was transient to an intermediate temporal regime by investigating cell dynamics at larger times $10 \mathrm{~s}<\tau<1 \mathrm{~h}$. To this end, we calculated the bulk long-time $\operatorname{MSD} \frac{\operatorname{MSD}^{\dagger}(\tau)}{\tau}=\frac{1}{\tau}\left\langle\left(r_{i}(t+\tau)-r_{i}(t)\right)^{2}\right\rangle_{t, i}$ divided by the lag time. We find that caging is persistent and continues up to $\tau \approx 300 \mathrm{~s}$ with an approximate exponent $\alpha \approx 0.5$ [Fig. 1(d)]; thereafter, the dynamics become shortly diffusive and then superdiffusive, which is not expected for colloidal systems. We hypothesized that cellular proliferation may cause radial cell movement. To assess this hypothesis, we split MSD ${ }^{\dagger}$ into radial and azimuthal components $\mathrm{MSD}_{r}=$ $\left\langle\left(\Delta \mathbf{r}_{i} \cdot \hat{\mathbf{e}}_{r}\right)^{2}\right\rangle, \mathrm{MSD}_{a}=\left\langle\left(\Delta \mathbf{r}_{i} \times \hat{\mathbf{e}}_{r}\right)^{2}\right\rangle$, respectively. We found that superdiffusive motion is limited to the radial part and thus probably growth induced, while the azimuthal part approaches a flat line indicative of diffusive behaviour. The contribution of cell division to the MSD can be predicted from the density equation [20]:

$$
\partial_{t} \phi=-\nabla \cdot\left(\mathbf{v}_{\lambda} \phi\right)+\lambda \phi,
$$

where $\lambda$ is the growth rate and $\mathbf{v}_{\lambda}$ the mass transport velocity field, with $\mathbf{v}_{\lambda}=v_{\lambda} \hat{\mathbf{e}}_{r}$ due to the spherical symmetry of aggregates. For $\lambda, \phi$ constant, Eq. (3) leads to a radial contribution of $\operatorname{MSD}_{\lambda}(\tau)=\left\langle r^{2}\right\rangle[1-\exp (\lambda \tau / 3)]^{2}$, with $\left\langle r^{2}\right\rangle$ the initial average squared radial position of cells. We previously showed $\lambda \approx 0.55 \mathrm{~h}^{-1}$ [18], which fits the experimental data well. To find out whether growth expansion fluidizes the cages, we compared radial and azimuthal exponents $\alpha_{a, r}$ for 20 colonies with varying growth rates, which were obtained by increasing the incubation time before recordings [18]. We found a positive correlation $\left(r_{\text {pearson }}=0.51, p=0.028\right)$ between the

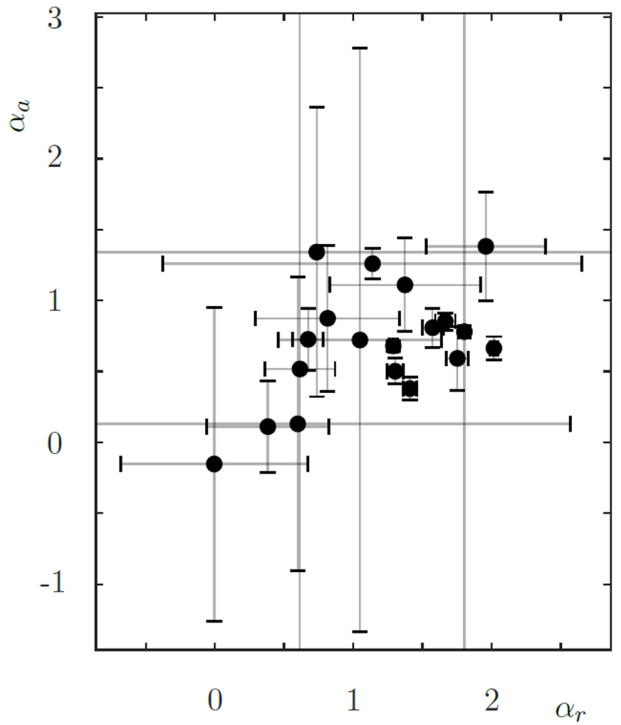

FIG. 3. Scaling exponent $\alpha_{a}$ of $\mathrm{MSD}_{a}$ plotted against the scaling exponent $\alpha_{r}$ of $\mathrm{MSD}_{r}$ at times $\tau>300 \mathrm{~s}$ for 20 colonies (strain $\mathrm{Ng} 194)$. Error bars represent fluctuations in time.

time-averaged exponent $\alpha_{r}$ (averaged over times $>500 \mathrm{~s}$ ) and $\alpha_{a}$, with $\alpha_{a} \approx 1$ diffusive at $\alpha_{r} \approx 2$ (Fig. 3), indicating that growth fluidizes colony dynamics at long timescales. The latter corresponds to the leading order exponent of the Taylor expansion of $\mathrm{MSD}_{\lambda}$. We conclude that caging effects govern the dynamics within the bulk of gonococcal colonies. For very short times, pilus retraction drives ballistic movement within cages created by neighboring cells. At times $\tau>5 \mathrm{~min}$, bacteria escape from the cages, consistent with fluidlike behaviour of the colonies.

\section{REDUCING THE VOLUME FRACTION CHANGES TRANSITIONS BETWEEN MOTILITY REGIMES}

Caging is critically affected by the volume fraction $\phi$. We addressed the question whether reduction of volume density delays or inhibits caging at intermediate timescales. We found that $\phi$ is lowest at the edge of the colony and continuously approaches a maximum $\phi_{M} \approx 0.5$ toward the center of the aggregate (Fig. 2). Based on this spatial heterogeneity, we analyzed cellular dynamics as a function of $\phi$. For times $\tau<3 \mathrm{~s}$, the VACF exhibits a similar qualitative behavior as in the bulk of the colony where $\phi \rightarrow \phi_{M}$ (Fig. 4). Quantitatively, $C_{v}(\phi, 0)$ decreased with increasing volume fractions. For cells at the aggregates surface, we find a maximal velocity $v_{0} \equiv\left[C_{v}(\phi \sim 0.1,0)\right]^{1 / 2} \approx 1.1 \mu \mathrm{m} / \mathrm{s}$, which is close to the value for pili-driven surface motility [21]. Correlation times of the initial decay slightly decrease toward the center of aggregates but appear roughly constant overall (Fig. 5). We then characterized motility regimes using Eq. (2). At lower volume fractions, the MSD transitions from a superdiffusive to a prolonged diffusive regime $[0.1<\tau<5$ s, Figs. 6(a) and 7(a)], followed by delayed caging $(\alpha<1)$. We hypothesized that the intermediate diffusive regime is akin to the long-time behavior of a persistent random walk with velocity $v_{0}$ and correlation time $\tau_{c}$ [5]. For $\tau_{c}$ constant and heterogeneous $v_{0}(\phi)$, 


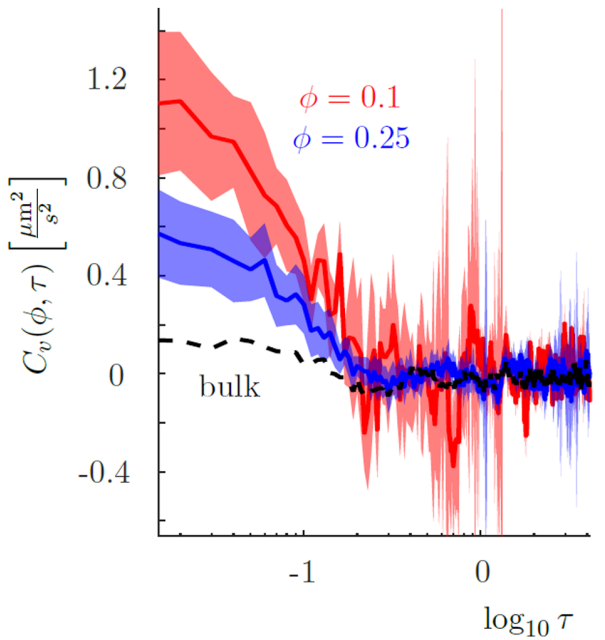

FIG. 4. Velocity autocorrelation function as a function of lag time and cell volume fraction for untreated cells (strain $\mathrm{Ng} 194$ ). Standard errors are marked by the shaded regions. The black dotted line indicates the bulk result.

the Fokker-Planck equation of the process has steady-state solution $D(\phi) \sim \phi^{-2}$ [22]. To test this prediction, we calculated experimental diffusion coefficients $D(\phi)=\frac{\mathrm{MSD}\left(\phi, \tau_{D}\right)}{4 \tau_{D}}$ at times $\tau_{D}(\phi)$ for which $\alpha \approx 1$, using both Eq. (2) and $\mathrm{MSD}^{\dagger}$. We find that diffusion coefficients follow a power law $D(\phi) \sim \phi^{\beta}$ in the range $0.15<\phi<0.5$, with $\beta=-1.96 \pm 0.04 \approx-2$ $\left(R^{2}>0.9\right)$, in agreement with the theory [Fig. 6(b)]. At lower densities, diffusivities approach values between 0.05 and 0.1 $\mu \mathrm{m}^{2} / \mathrm{s}$, which depend on the maximum achievable retraction velocity at minimal load. For $\phi \approx \phi_{M}$, diffusivities drop sharply with large error bars and further deviate from the power law behavior due to missing contributions of higher order density correlations in the derivation of the Fokker-Planck equation.

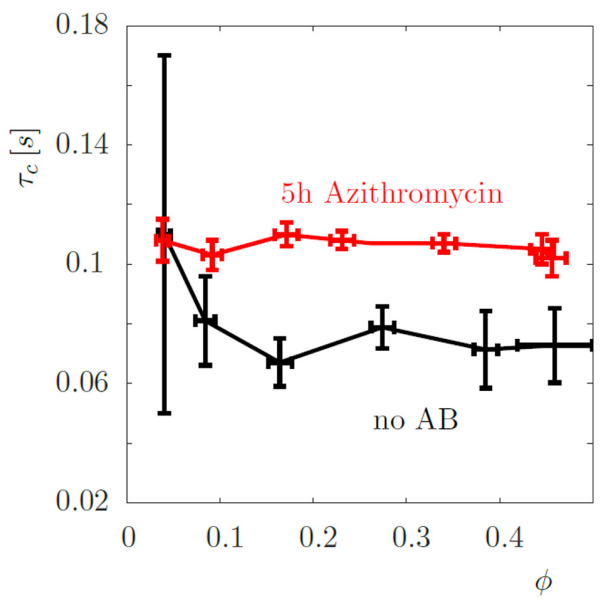

FIG. 5. Correlation time $\tau_{c}$ of the initial decay of the velocity autocorrelation function $C_{v}$ as a function of the volume fraction $\phi$ of cells for untreated cells (black curve) and cells treated for $5 \mathrm{~h}$ with azithromycin (red curve). Correlation times are extracted using exponential fits for $\tau<0.5 \mathrm{~s}\left(R^{2}\right.$ between 0.7 and 0.9 for untreated cells, and between 0.8 and 0.9 for treated cells). Error bars mark standard errors over 40 colonies.
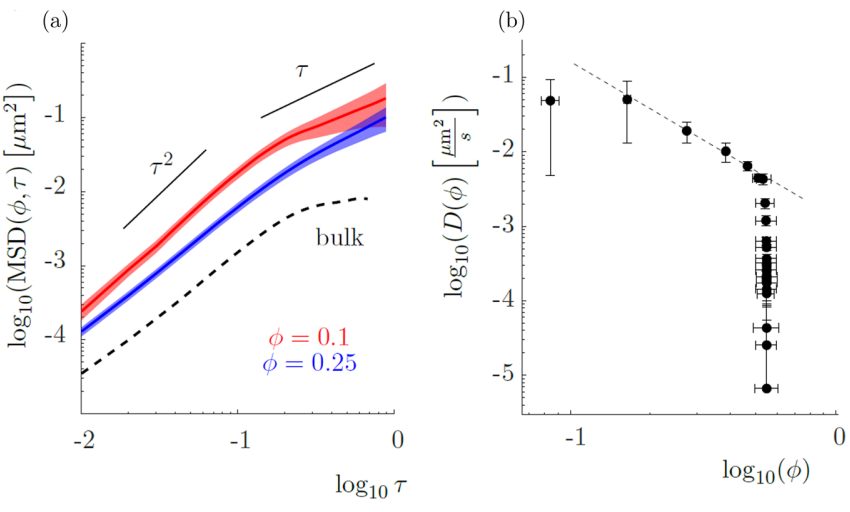

FIG. 6. Single-cell dynamics in colonies as a function of $\phi$, averaged over 40 colonies ( $\mathrm{Ng} 194)$. (a) Mean-squared displacement $[\operatorname{MSD}(\phi, \tau)]$ obtained from Eq. (2) for lower values of $\phi$. (b) Diffusivities as a function of $\phi$. The black dotted line is a power law fit $\phi^{\beta}$ for $0.15<\phi<0.5$, which yields $\beta=-1.96 \pm 0.04$. Shaded regions and error bars are standard errors.

Because $D(\phi \approx 0.1) \ll D_{\text {thermal }}=\mu k_{\mathrm{B}} T$, where $\mu=\frac{1}{6 \pi \eta a}$ (cell diameter $a \approx 0.5 \mu \mathrm{m}$ ) and $T \approx 300 \mathrm{~K}$, the effective dynamic viscosity $\eta$ is two orders of magnitude larger than for thermalized colloidal spheres of the same size. The resulting larger drag effectively freezes cells in place under the control of the retractive action of the pili [13]. In summary, at reduced volume fractions, we observe a transition between the ballistic motion of T4P retractions to diffusive motion at intermediate timescales. In this regime, the diffusion coefficient scales with the volume fraction like $D(\phi) \sim \phi^{-2}$. (a)

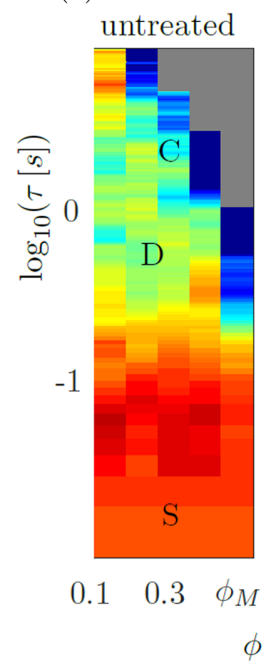

(b)

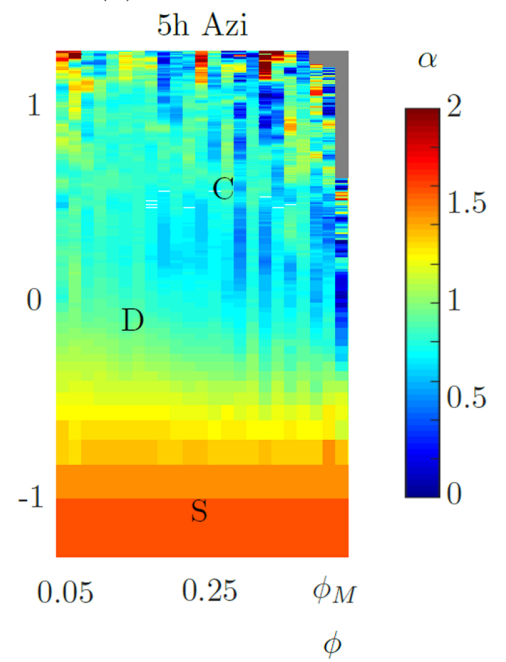

FIG. 7. Exponent $\alpha$ of the mean-square displacement data as a function of volume fraction $\phi$ and lag time $\tau$ for (a) untreated (strain $\mathrm{Ng} 194)$ and (b) treated cells (5 h azithromycin at $100 \times$ MIC, strain Ng150). $S, D, C$ denote superdiffusive, diffusive, and subdiffusive (caged) regimes, respectively. In gray areas, the standard error is larger than the exponent. For treated cells, the time axis is shifted downward. 

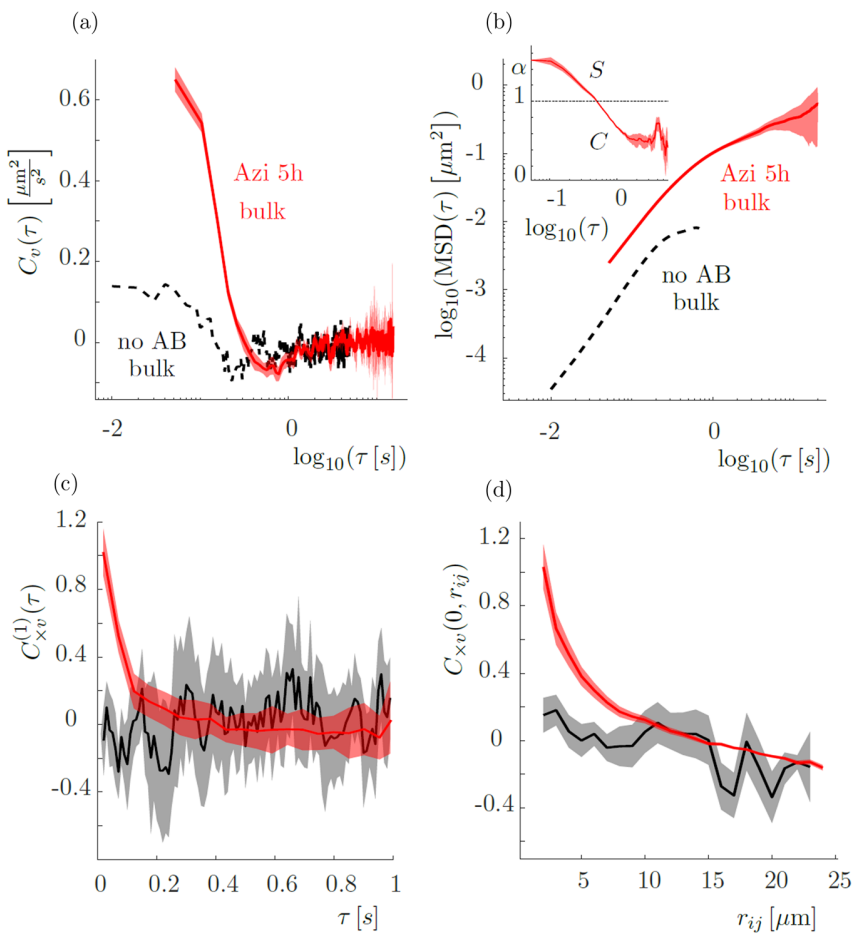

FIG. 8. Single-cell dynamics and cross-correlations in treated colonies (40 colonies evaluated, strain $\mathrm{Ng} 150$ ). (a) $C_{v}(\tau)$ as a function of lag time $\tau$ between 0.01 and $10 \mathrm{~s}$ (red curve). The black dotted line is the result for untreated cells. (b) Bulk mean-square displacement as a function of time, with motility exponent $\alpha$ (inlet). (c) First-shell cross-correlation $C_{\times v}^{(1)}$ between 0.01 and $1 \mathrm{~s}$ for untreated (black curve) and treated cells (red curve). (d) Spatial correlation $C_{\times v}$ as a function of cell pair distance $r_{i j}$ for untreated (black curve) and treated cells (black curve). Shaded regions represent standard errors.

\section{REDUCING T4P-T4P ATTRACTION BY ANTIBIOTIC TREATMENT FLUIDIZES THE COLONIES AND INTRODUCES LONG-RANGE INTERACTIONS}

We expect that attractive interactions modulate cell dynamics and caging effects. In gonococcal colonies, T4P-T4P binding governs the attractive interaction between cells. We reported previously that treatment with the antibiotic azithromycin reduced the T4P-mediated attractive force between neighboring cells, yet $\mathrm{T} 4 \mathrm{P}$ retraction is still active, and diffusion constants are enhanced at a timescale of $\sim 1 \mathrm{~s}$ [17]. Treatment does not dissolve the colonies, and cell mortality is negligible. Here, we treated colonies (strain $\mathrm{Ng} 150$, see Table I) with azithromycin to address the effect of attractive force on cellular dynamics within colonies. Qualitatively, the overall dynamics in terms of the VACF and MSD in the bulk of the colony is like the untreated phenotype [Figs. 8(a) and 8(b)]. The shape of the VACF is conserved, and we retrieve the persistent region with backscattering. The MSD and its exponent $\alpha$ transitions from a superdiffusive through a diffusive toward a subdiffusive regime. Transitions, however, are strongly delayed to $\sim 0.4-0.5 \mathrm{~s}$. In line with previous studies [17], the motility in treated colonies is increased, which we explain by a higher velocity modulus, longer correlation times $\left(\tau_{c}^{\text {Azi }} \approx 0.11 \pm 0.01 \mathrm{~s}\right.$, Fig. 5) and by delayed backscattering and caging, all in line with

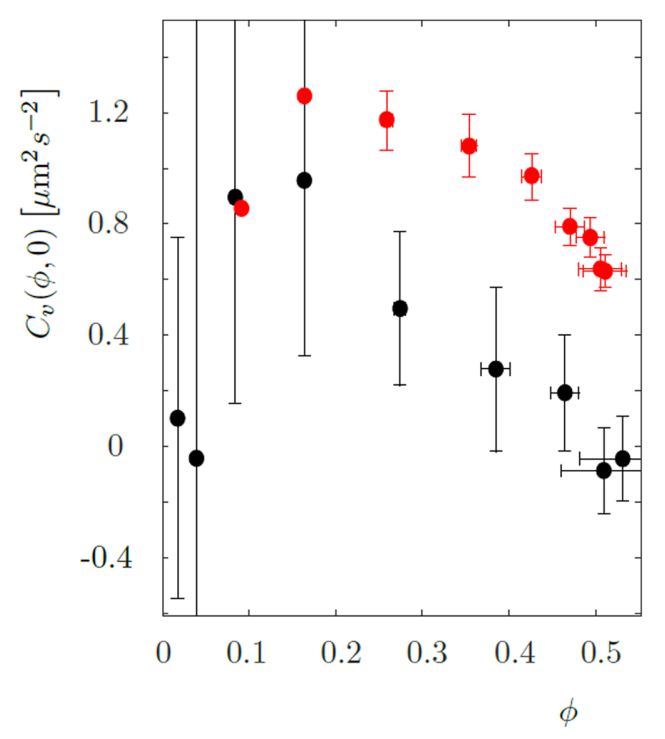

FIG. 9. Velocity modulus $C_{v}(\phi, 0)$ as a function of volume fraction for treated (red, strain $\mathrm{Ng} 150,5 \mathrm{~h}$ azithromycin at 100-fold MIC) and untreated cells (black, strain $\mathrm{Ng} 194$ ). Error bars represent standard errors.

an increased cell-cell distance and reduced attractive force between cells. For times $\tau \geqslant 0.5 \mathrm{~s}$ beyond the initial persistence, the negative VACF decays asymptotically toward zero. We further measured the impact of varying volume fractions for treated colonies. The modulus $C_{v}(\phi, 0)$ decays as $\phi$ increases and is overall marginally higher than for untreated cells (Fig. 9), with $C_{v}(\phi \rightarrow 0.1,0) \approx 1.2 \mu \mathrm{m}^{2} / \mathrm{s}^{2}$, in agreement with the velocity of single $\mathrm{T} 4 \mathrm{P}$ retraction. At lower volume fractions, the exponent $\alpha(\phi, \tau)$ shows a delayed intermediate diffusive regime before the dynamics turn subdiffusive at later times [Fig. 7(b)]. In contrast to

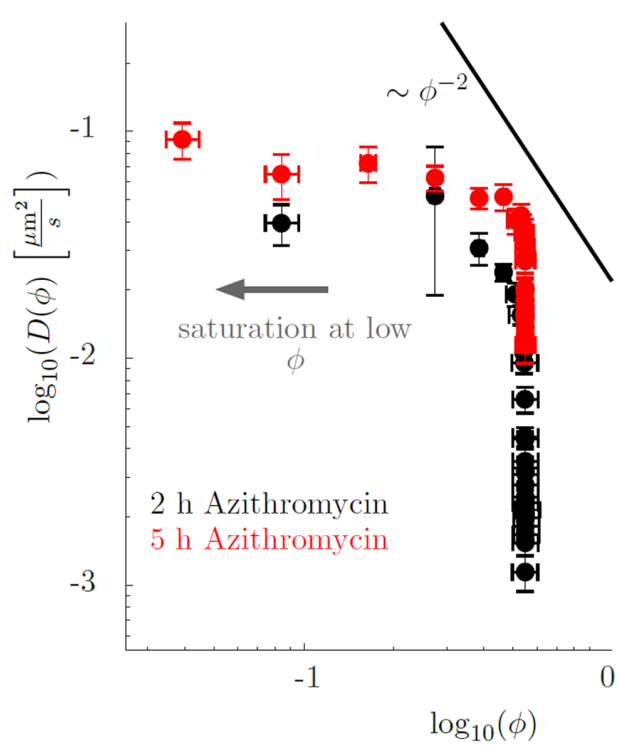

FIG. 10. Intermediate diffusivities as a function of volume fraction $\phi$. Red curve: $5 \mathrm{~h}$ treatment with azithromycin. Black curve: $2 \mathrm{~h}$ treatment. In both cases, we used the $\mathrm{Ng} 150$ strain and an azithromycin concentration of $6.4 \mu \mathrm{g} / \mathrm{mL}(\mathrm{MIC} \times 100)$. Full line is $D(\phi) \sim \phi^{-2}$. Error bars represent standard errors. 
(a)
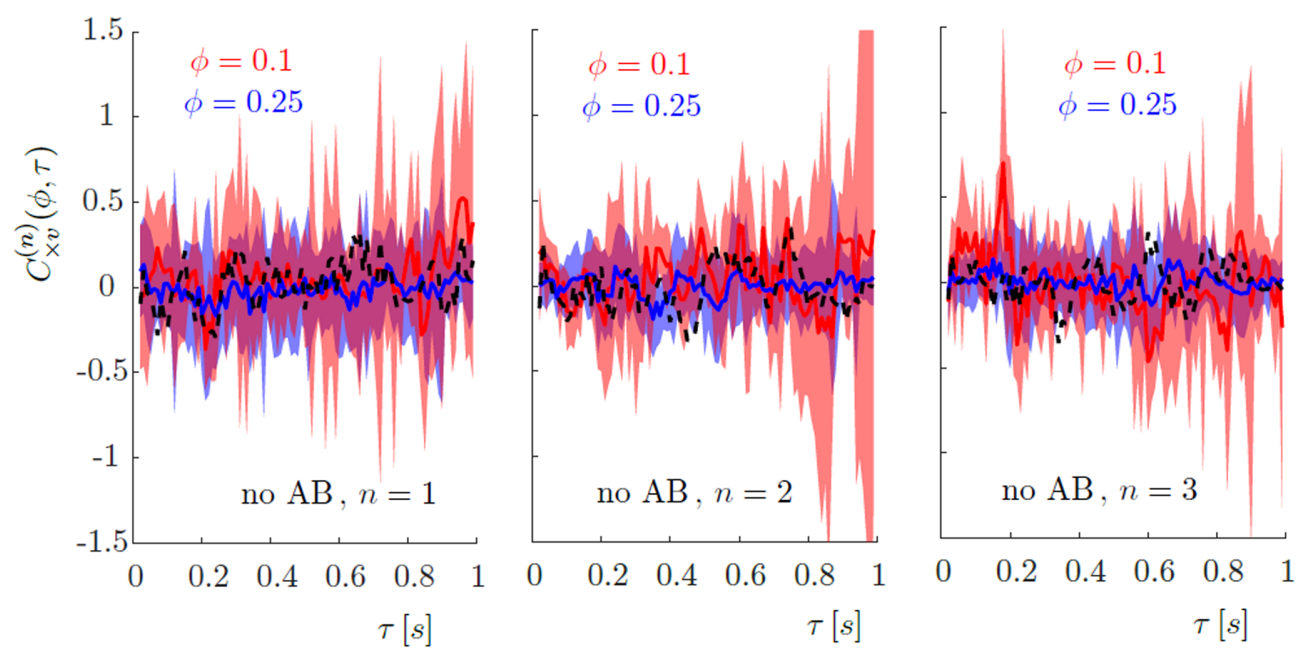

(b)
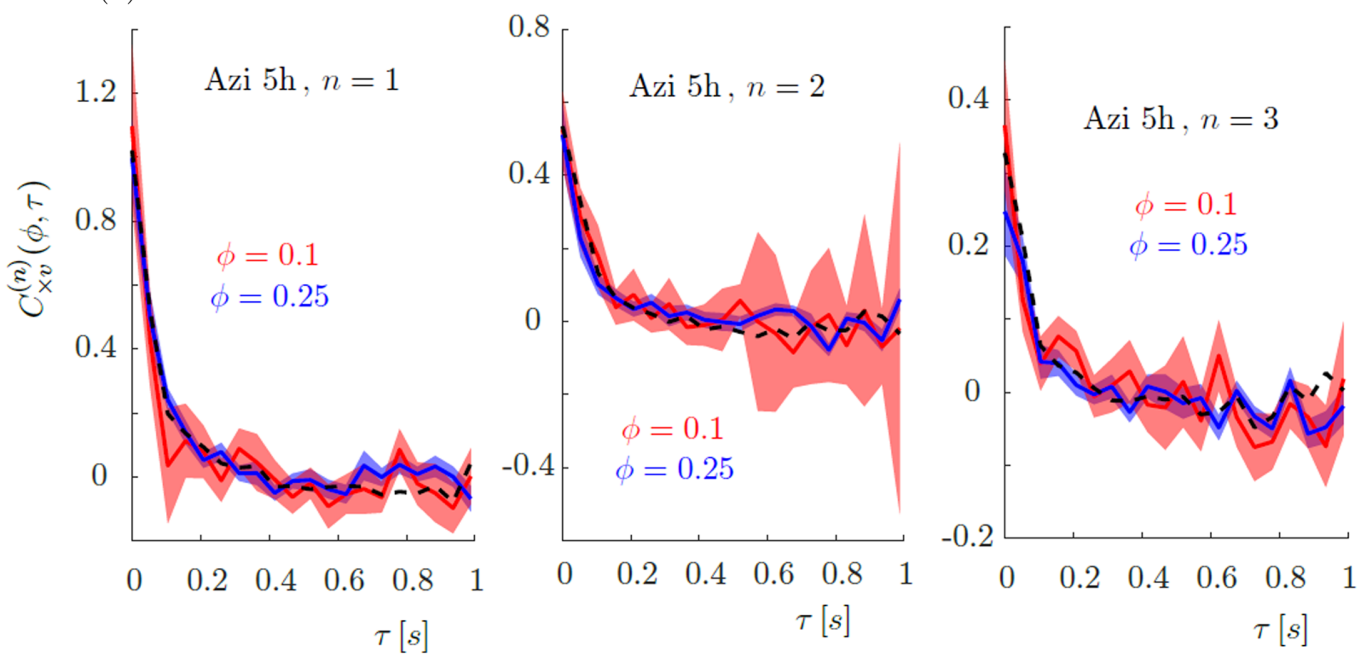

FIG. 11. Velocity cross-correlations as a function of lag time $\tau$ and volume fraction $\phi$. (a) Velocity cross-correlation function (VCCF) for untreated cells (strain Ng194) and for the first three neighbor shells. The black dotted lines are bulk results for $\phi \rightarrow \phi_{M}$. (b) VCCF for treated cells (5 h azithromycin at $100 \times \mathrm{MIC}$, strain $\mathrm{Ng} 150$ ) and for the first three neighbor shells. Shaded regions indicate standard errors.

the no $\mathrm{AB}$ condition, intermediate experimental diffusivities $D(\phi)$ for treated cells do not follow an inverse square law, most likely because the decay of the velocity modulus $C_{v}(\phi, 0)$ at intermediate volume fractions is less pronounced. Instead, $D(\phi)$ slightly decreases as $\phi$ increases and sharply drops for $\phi \approx \phi_{M}$. This further indicates that short- and intermediate-time dynamics in treated colonies approach an upper motility limit, in line with a finite retraction velocity of the pilus motor at small loads [11]. We additionally measured diffusivities for cells treated for $2 \mathrm{~h}$ with azithromycin at the same concentration. In this case, we observe a weaker increase of intermediate diffusivities, which retains the functional power law relationship for $0.28<\phi<0.5$, see Fig. 10 (fit exponent: $-1.75 \pm 0.4$ ).

Next, we asked whether pairwise pilus retractions cause coupling of the motion of nearby cells. We calculated the velocity cross-correlation function (VCCF), which measures the velocity correlation of bulk cells $i, j$ at different distances $r_{i j}$ as a function of $\tau$. We used the normalized form [23]:

$$
C_{\times v}^{(n)}(\tau)=\frac{1}{N}\left\langle\mathbf{v}_{i}(t+\tau) \cdot \mathbf{v}_{j}(t)\right\rangle_{t, r_{i j} \in(n)},
$$

with $N=C_{v}\left(\phi \rightarrow \phi_{M}, 0\right)$. The index (n) denotes neighbor shells at distances $r_{i j}$ equal to integer multiples of the first maximum $r_{0}$ of the radial distribution function $g^{(2)}\left(r_{i j}\right)$ [13]. As such, the cross-correlation captures the degree of comotion for pairs of cells at a certain distance from each other. The VCCF was calculated for untreated cells (no AB) and cells treated with azithromycin $(\mathrm{AB})$. We used $r_{0}^{\mathrm{noAB}}=1.3 \mu \mathrm{m}$ and $r_{0}^{\mathrm{AB}}=1.7 \mu \mathrm{m}$ [17] and set a shell width of $r_{0}$. Within the bulk of untreated colonies and for $n=1$, we find that cross-correlations fluctuate around zero at all times [Fig. 8(c)]. We thus argue that nearby fought tug-of-wars are independent of each other, even though they share pilus connections. Similar patterns appeared for the second and third shell and at lower volume fractions [Fig. 11(a)]. Remarkably, we observe strong temporal cross-correlations for cells under the 


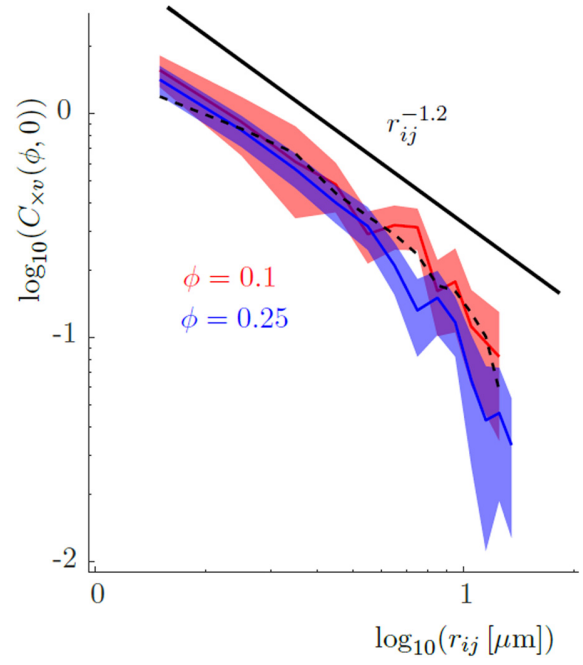

FIG. 12. $C_{\times v}(\phi, 0)$ as a function of pair particle distance $r_{i j}$ for treated colonies ( $5 \mathrm{~h}$ azithromycin, strain $\mathrm{Ng} 150$, averaged over 40 colonies). Colors indicate volume fractions, with the black dotted line showing results for the bulk of colonies. The full black line shows the best power law fit for curves averaged over $\phi$. Shaded regions indicate standard errors.

$\mathrm{AB}$ condition. In the first shell and within the bulk, the initial VCCF is close to unity. The subsequent decay is well captured by an exponential function with characteristic decay time of $\tau_{\text {cross }}=0.08 \pm 0.03 \mathrm{~s}\left(R^{2} \approx 0.9\right)$, which is close to the correlation time $\tau_{c}^{\text {Azi }}$. Coupling is also present for higher shells [Fig. 11(b)] and for lower volume fractions, albeit with reduced velocity moduli. To quantify the spatial extent of collective motion, we calculated the correlation length $r_{c}$ at which $C_{\times v}^{(n)}\left(\phi \rightarrow \phi_{M}, 0\right)$ as a function of $r_{i j}$ (in bins of $1 \mu \mathrm{m}$ ) has decreased to $\frac{1}{2}$ its initial value. The correlation in the bulk decays asymptotically to zero over a distance of roughly $10 \mu \mathrm{m}$, with $r_{c} \approx 3.5 \pm 0.3 \mu \mathrm{m}$, see Fig. 8(d). We note that the decay distance is much larger than the average pilus length of $1 \mu \mathrm{m}$ [11]. We hypothesize that long-range hydrodynamic interactions and a diluted pili network may be responsible for the observed comobility of cells. At higher cell number densities (no AB condition), the creeping flow created by the retraction events is screened by nearby cells with no-slip surfaces [24]. At smaller densities ( $\mathrm{AB}$ condition), screening is reduced, and the pilus retractions create long-ranged flow fields. For point forces, the leading-order coupling is predicted to decay with a power law exponent of $\beta=-1$ [25], which is close to the observed exponent $\beta=-1.2 \pm 0.2$ (averaged over volume fractions, see Fig. 12) obtained by fitting curves to power laws in the range $0<r_{i j}<7 \mu \mathrm{m}$.

\section{CONCLUSIONS}

We investigated the dynamics of aggregated $N$. gonorrhoeae cells on five timescales at varying volume fractions and attractive interactions. The short time behavior of clustered bacteria is dominated by ballistic pilus retractions and contact backscattering. Cells transition from a superdiffusive to a diffusive or caged regime, which is fluidized by the growth-induced expansion of colonies at $\sim 300 \mathrm{~s}$. Reducing $\phi$ and modulating attractive interactions leads to delayed caging, which increases motility and may help cells disperse more easily under stressors like oxygen deprivation [26]. In this paper, we thus shed light on dynamical heterogeneities within early biofilms, which may be crucial for cell differentiation at later times [18]. Recently, we described fluidlike shape relaxation dynamics of gonococcal colonies at a timescale of minutes [17]. This behavior is in excellent agreement with cell dynamics found here. At this timescale, bacteria escape from their cages because active T4P dynamics and growth enable fluidlike behavior. Further, measured and calculated intermediate diffusivities are in line with previous work $[14,17]$. Motility in $N$. gonorrhoeae aggregates shows interesting parallels to equilibrium and nonequilibrium systems. The regime transitions for single-cell dynamics mirror the behavior of thermalized simple liquids at effective temperatures of a few kelvin [27]. At the same time, the dependence of $v_{0}$ on the volume fraction is typically encountered in dense active matter suspensions of monopole swimmers, which aggregate by a different mechanism: For such particles, repeated collisions between tumbles increase the time it takes a cell to move a certain distance, such that effectively, the propulsion speed decreases with the number of collisions and thus the volume fraction [9]. This leads to an accumulation of swimmers in high-density regions, triggering a positive feedback loop leading to aggregation through a process called motility-induced phase separation [9]. Although this collision picture is hardly applicable here, cell dynamics inside neighbor cages are well captured by a persistent random walk with heterogeneous swimming velocities whose correlation time can be inferred from the cell-cell distance. Alternatively, one could ignore the initial persistent regime and model colonies as a hard sphere colloidal system with multiplicative noise in the Ito interpretation, where cells accumulate in regions of low diffusivity [28]. We argue that the nonhomogeneous velocity modulus can be explained by variations of the hydrodynamic mobilities or by a varying density of the network of pili connections. For the former, it is known that drag forces are stronger in the presence of no-slip boundaries [29,30], which in this case are realized by the surface of other (on average) immobile neighboring cells. For the latter, we imagine that at higher cell volume fractions, more pili connections effectively hinder proper pilus retractions by increasing the load. In the future, we will thus try to bridge the gap between the microscopic tug-of-war pilus dynamics and the resulting cell body dynamics described here.

\section{ACKNOWLEDGMENTS}

We thank T. Kranz and H. Löwen for helpful discussions. Funding is provided by the Center for Molecular Medicine Cologne and the Deutsche Forschungsgemeinschaft by Grant No. MA3898.

\section{APPENDIX: MATERIALS AND METHODS}

\section{Bacterial strains and growth conditions}

Gonococcal base agar was made from $10 \mathrm{~g} / \mathrm{L}$ dehydrated agar (BD Biosciences, Bedford, MA), $5 \mathrm{~g} / \mathrm{L} \mathrm{NaCl}$ (Roth, Darmstadt, Germany), $4 \mathrm{~g} / L \mathrm{~K}_{2} \mathrm{HPO}_{4}$ (Roth), $1 \mathrm{~g} / \mathrm{L}$ 
$\mathrm{KH}_{2} \mathrm{PO}_{4}$ (Roth), $15 \mathrm{~g} / \mathrm{L}$ Proteose Peptone No. 3 (BD Biosciences), $0.5 \mathrm{~g} / \mathrm{L}$ soluble starch (Sigma-Aldrich, St. Louis, MO), and supplemented with $1 \%$ IsoVitaleX (IVX): $1 \mathrm{~g} / \mathrm{L}$ D-glucose (Roth), $0.1 \mathrm{~g} / \mathrm{L}$ L-glutamine (Roth), $0.289 \mathrm{~g} / \mathrm{L}$ L-cysteine-HCL $\times \mathrm{H}_{2} \mathrm{O}$ (Roth), $1 \mathrm{mg} / \mathrm{L}$ thiamine pyrophosphate (Sigma-Aldrich), $0.2 \mathrm{mg} / \mathrm{LFe}\left(\mathrm{NO}_{3}\right)_{3}$ (Sigma-Aldrich), $0.03 \mathrm{mg} / \mathrm{L}$ thiamine $\mathrm{HCl}$ (Roth), $0.13 \mathrm{mg} / \mathrm{L}$ 4-aminobenzoic acid (Sigma-Aldrich), $2.5 \mathrm{mg} / \mathrm{L} \beta$-nicotinamide adenine dinucleotide (Roth), and $0.1 \mathrm{mg} / \mathrm{L}$ vitamin B12 (Sigma-Aldrich). GC medium is identical to the base agar composition but lacks agar and starch. All bacterial strains (Table I) are derivatives of strain $N$. gonorrhoeae MS11. Strain $w t^{*}$ green $\Delta$ pilT $(\mathrm{Ng} 231)$ was generated as follows. Strain $w t^{*}$ green $(\mathrm{Ng} 194)$ was transformed with genomic DNA from strain $\Delta p i l T$ (Ng178) [13]. Transformants were selected on agar plates containing $10 \mu \mathrm{g} / \mathrm{mL}$ chloramphenicol.

\section{Microscopy}

Overnight cultures of the sfgfp expressing strain $\mathrm{Ng} 194$ were suspended in fresh GC + IVX medium at an $O D$ of 0.1 unless noted otherwise. After an additional hour of incubation, cells were loaded into a microfluidic flow chamber (Ibidi Luer $0.8 \mathrm{~mm}$ channel height + Ibitreat) which was previously coated with a Poly-L-Lysine (Sigma, Cat. No. P4832, $50 \mu \mathrm{g} / \mathrm{mL}$ ) solution for $2 \mathrm{~h}$. The flow chamber was continuously supplemented with medium using a peristaltic pump (model 205U; Watson Marlow, Falmouth, United Kingdom). Images were acquired using an inverted microscope (Ti-E, Nikon) equipped with a spinning disc confocal unit (CSU-X1, Yokogawa) and a 100×, 1.49 NA, oil immersion objective lens (excitation wavelength: $488 \mathrm{~nm}$ ). Under AB conditions, $\mathrm{Ng} 150$ colonies were additionally supplied with azithromycin (Hello Bio, $6.4 \mu \mathrm{g} / \mathrm{mL}$ ) at $100 \times$ MIC for 2 and $5 \mathrm{~h}$ before imaging. Moreover, the fluorescent dye Syto 9 (final concentration: $3 \mu \mathrm{M}$ ) was added to the medium because it yields a better fluorescence signal than the $w t^{*}$ strain under AB conditions. Short timescale dynamics were captured at a frequency of 80 to $100 \mathrm{~Hz}$ (no AB conditions) and $20 \mathrm{~Hz}$ (AB condition) and a laser intensity of $1 \%$. For the long timescale dynamics (no $\mathrm{AB}$ condition), the acquisition frequency was changed to $0.1 \mathrm{~Hz}$ to avoid photodamage and photobleaching over the recording time of $1 \mathrm{~h}$. We then used the ImageJ Plugin TrackMate to track bacterial cells in space and time.

\section{Volume fraction of cells as a function of depth}

Volume fractions were obtained by counting cells in bins of width $\Delta d=0.5 \mu \mathrm{m}$ at a distance $r$ from the center of mass with position $r_{\mathrm{COM}}=\left\langle r_{i}\right\rangle_{i}(i$ : cell index) and dividing the count by the cylindrical volume $V(r)=2 \pi r \Delta d \Delta z$. We set $\Delta z:=600 \mathrm{~nm}$ as the capture resolution of the microscope in the direction perpendicular to the image plane. The volume fraction occupied by cells is $(r)=\frac{N}{V(r)} \frac{3 \pi a_{\text {cell }}^{3}}{4}$. From individual cell signal profiles, we obtain radii of $a_{\text {cell }}^{\text {no } \mathrm{AB}} \approx 0.47 \mu \mathrm{m}$ and $a_{\text {cell }}^{5 \mathrm{~h} \text { Azi }} \approx 0.69 \mu \mathrm{m}$, in line with previously measured values [17]. The volume fraction profile is depicted in Fig. 2. It is highest in the bulk of the colonies and decreases toward the condensate-gas/vacuum interface. We fitted the curves to instantons (sharp interfaces with finite profile width $e$ ) [31], $\phi_{\text {fit }}(d)=\frac{\phi_{M}}{2}\left[1+\tanh \left(\frac{d}{e}\right)\right]$, from which we further obtained the colony radius $R$. Here, $\phi_{M}$ is the saturation volume fraction deep in the colony, and $d$ is the inward distance starting from the edge of the colonies, such that $\phi(d=0)=\frac{1}{2} \phi_{M}$. We find $\phi_{M} \approx 0.515, e_{\mathrm{no} \mathrm{AB}} \approx 1.2 \mu \mathrm{m}, e_{5 \mathrm{~h} \mathrm{Azi}} \approx 2.6 \mu \mathrm{m}$. Because $\Delta z$ could be overestimated, it is possible that $\phi_{M}$ is closer to the random close packing fraction of spheres.

\section{Correction for colony translation and rotation}

Individual velocities can be written as $v_{i}=v_{\text {cell }}+v_{\mathrm{CT}}+$ $v_{\mathrm{CR}}$, where $v_{\text {cell }}$ captures individual cell dynamics, and $v_{\mathrm{CT}, \mathrm{CR}}$ represent rigid body translation (CT) and rotation (CR) of the whole colony. In addition to coating of the flow chambers, we minimized these in two additional ways: Time series are stabilized using the Fiji-plugin StackReg. Second, we subtracted the ensemble-averaged velocity $\left\langle v_{i}\right\rangle_{i}$ (assumed to be equal to $v_{\mathrm{CT}}$ ) and the angular velocity $\left\langle w_{i}\right\rangle_{i} \times r_{i}$ (assumed to be equal to $v_{\mathrm{CR}}$ ) from $v_{i}$ (measured velocity of cell $i$ ) and corrected positions by integration. The proper functioning of this last step was tested by simulating dummy colonies with (a) white noise translation and rotation and (b) constant translational and rotational velocities.

\section{Static displacement errors}

We checked first for pixel bias/locking in the 2D particle positions and found none. Nevertheless, we corrected for possible irregularities in the metapixel distribution by applying the SPIFF-correction algorithm [32]. Particle positions are plagued by an uncorrelated stochastic error $\varepsilon$ of unknown strength, i.e., $\mathbf{r}_{i}(t)=\widetilde{\mathbf{r}}_{i}(t)+\boldsymbol{\varepsilon}\left(t, r_{i}\right)$, where . marks the true position, and we assume $\langle\boldsymbol{\varepsilon}\rangle=0,\left\langle\boldsymbol{\varepsilon}\left(t_{1}, \mathbf{r}_{1}\right) \cdot \boldsymbol{\varepsilon}\left(t_{2}, \mathbf{r}_{2}\right)\right\rangle=$ $g \delta\left(t_{2}-t_{1}\right) \delta\left(\mathbf{r}_{2}-\mathbf{r}_{2}\right)$. To leading order, this error propagates into the VACF as $C_{v}(\tau)=\left\langle\frac{\Delta \boldsymbol{r}_{i}(t)}{\Delta t} \cdot \frac{\Delta \boldsymbol{r}_{i}(t+\tau)}{\Delta t}\right\rangle=\widetilde{C}_{v}(\tau)+$ $C_{\varepsilon}(\tau)$, where $C_{\varepsilon}(0)=2\left\langle\left(\frac{\Delta \varepsilon}{\Delta t}\right)^{2}\right\rangle, C_{\varepsilon}(\Delta t)=-\left\langle\left(\frac{\Delta \varepsilon}{\Delta t}\right)^{2}\right\rangle, C_{\varepsilon}(\tau>$ $\Delta t)=0$. Thus,

$$
\widetilde{C}_{v}(\tau)=C_{v}(\tau) \text { for } \tau>\Delta t
$$

We thus removed the first and second elements and recovered $\widetilde{C}_{v}(\Delta t)$ by calculating when possible the slope at later times and extrapolating backward. The error propagation into the MSD is given by $\operatorname{MSD}(\tau)=\widehat{\operatorname{MSD}}(\tau)-2\left(\Delta t^{2}\right) C_{\varepsilon}(\Delta t)$. For $d$ large (deep in the colony), we find that $\left|C_{\varepsilon}(\Delta t)\right| \gg\left|\widetilde{C}_{v}(\Delta t)\right|$ and thus assume $\widehat{\operatorname{MSD}}(\tau) \approx \operatorname{MSD}(\tau)+2(\Delta t)^{2} C_{v}(\Delta t)$. Because the error is larger than $\widetilde{\mathrm{MSD}}$ for times $<0.5 \mathrm{~s}$, we only used this form for $\mathrm{MSD}^{\dagger}$ and the determination of the intermediate diffusivities. For the radial and azimuthal part of $\mathrm{MSD}^{\dagger}$, the correction is half as large. The leading order error term in the first and second elements of velocity cross-correlations (at $\tau=0$ and $\tau=\Delta t$ ) arises from the boundary condition of vanishing mean translational and rotational velocities and scales as $N^{-1}$, where $N$ is the number of tracked cells at every timepoint. For $C_{\times v}^{(n)}(\tau)$, we thus removed again the first two elements. For $C_{\times v}\left(\tau \rightarrow 0, r_{i j}\right)$ as a function of 
the pair distance $r_{i j}$, it can be shown that the error is equal to $\frac{2}{N}\left[\frac{3}{2} C_{\varepsilon}\left(r_{i}, \Delta t\right)+\frac{3}{2} C_{\varepsilon}\left(r_{j}, \Delta t\right)-\frac{9}{4} \frac{2}{R^{2}} \int_{0}^{R} r C_{\varepsilon}(r, \Delta t) d r\right]$, where $C_{\varepsilon}(r, \Delta t)$ is the error of the VACF at $\tau=\Delta t$ and for different distances $r$ to the center of mass of the colony. We again assumed $C_{\varepsilon}(\Delta t) \approx C_{v}(\Delta t)$ for untreated cells deep in the colony and corrected the VCCF accordingly.
[1] E. Zaccarelli and W. C. K. Poon, Colloidal glasses and gels: the interplay of bonding and caging, Proc. Natl Acad. Sci. USA 106, 15203 (2009).

[2] P. M. Reis, R. A. Ingale, and M. D. Shattuck, Caging Dynamics in a Granular Fluid, Phys. Rev. Lett. 98, 188301 (2007).

[3] L. Berthier, E. Flenner, and G. Szamel, Glassy dynamics in dense systems of active particles, J. Chem. Phys. 150, 200901 (2019).

[4] D. L. Higashi, S. W. Lee, A. Snyder, N. J. Weyand, and A. Bakke, Dynamics of Neisseria gonorrhoeae attachment: microcolony development, cortical plaque formation, and cytoprotection, Infect. Immun. 75, 4743 (2007).

[5] C. Holz, D. Opitz, L. Greune, R. Kurre, M. Koomey, M. A. Schmidt, and B. Maier, Multiple Pilus Motors Cooperate for Persistent Bacterial Movement in Two Dimensions, Phys. Rev. Lett. 104, 178104 (2010).

[6] A. J. Merz, M. So, and M. P. Sheetz, Pilus retraction powers bacterial twitching motility, Nature (London) 407, 98 (2000).

[7] J. M. Skerker and H. C. Berg, Direct observation of extension and retraction of type IV pili, Proc. Natl Acad. Sci. USA 98, 6901 (2001).

[8] G. Gompper, R. G. Winkler, T. Speck, A. Solon, C. Nardini, F. Peruani, H. Löwen, R. Golestanian, U. B. Kaupp, L. Alvarez, T. Kiørboe, E. Lauga, W. C. K. Poon, A. DeSimone, S. MuiñosLandin, A. Fischer, N. A. Söker, F. Cichos, R. Kapral, P. Gaspard et al., The 2020 motile active matter roadmap, J. Phys. Condens. Matter 32, 193001 (2020).

[9] M. E. Cates and J. Tailleur, Motility-induced phase separation, Annu. Rev. Condens. Matter Phys. 6, 219 (2015).

[10] G. Gonnella, D. Marenduzzo, A. Suma, and A. Tiribocchi, Motility-induced phase separation and coarsening in active matter, C.R. Phys. 16, 316 (2015).

[11] R. Marathe, C. Meel, N. Schmidt, L. Dewenter, R. Kurre, L. Greune, M. A. Schmidt, M. J. I. Müller, R. Lipowsky, B. Maier, and S. Klumppand, Bacterial twitching motility is coordinated by a two-dimensional tug-of-war with directional memory, Nat. Commun. 5, 3759 (2014).

[12] B. Maier, How physical interactions shape bacterial biofilms, Annu. Rev. Biophys. 50, 401 (2021).

[13] A. Welker, T. Cronenberg, R. Zoellner, C. Meel, K. Siewering, N. Bender, M. Hennes, E. R. Oldewurtel, and B. Maier, Molecular Motors Govern Liquidlike Ordering and Fusion Dynamics of Bacterial Colonies, Phys. Rev. Lett. 121, 118102 (2018).

[14] D. Bonazzi, V. Lo Schiavo, S. Machata, I. Djafer-Cherif, P. Nivoit, V. Manriquez, H. Tanimoto, J. Husson, N. Henry, H. Chaté, R. Voituriez, and G. Duménil, Intermittent pili-mediated forces fluidize Neisseria meningitidis aggregates promoting vascular colonization, Cell 174, 143 (2018).

[15] W. Pönisch, K. B. Eckenrode, K. Alzurqa, H. Nasrollahi, C. Weber, V. Zaburdaev, and N. Biais, Pili mediated intercellular forces shape heterogeneous bacterial microcolonies prior to multicellular differentiation, Sci. Rep. 8, 16567 (2018).

[16] H.-S. Kuan, W. Poenisch, F. Juelicher, and V. Zaburdaev, Continuum Theory of Active Phase Separation in
Cellular Aggregates, Phys. Rev. Lett. 126, 018102 (2021).

[17] T. Cronenberg, M. Hennes, I. Wielert, and B. Maier, Antibiotics modulate attractive interactions in bacterial colonies affecting survivability under combined treatment, PLoS Pathog. 17, e1009251 (2021)

[18] A. Welker, M. Hennes, N. Bender, T. Cronenberg, G. Schneider, and B. Maier, Spatio-temporal dynamics of growth and death within bacterial colonies, Biophys. J. 120, 3418 (2021).

[19] J. R. Dorfman, H. van Beijeren, and T. R. Kirkpatrick, Contemporary Kinetic Theory of Matter (Cambridge University Press, Cambridge, 2021).

[20] H. Raphael, G. Alexander, W. C. K. Dietrich, G. Gerhard, and E. Jens, Quantitative modelling of nutrient limited growth of bacterial colonies in microfluidic cultivation, J. R. Soc. Interface 15, 20170713 (2018).

[21] J. Eriksson, O. S. Eriksson, L. Maudsdotter, O. Palm, J. Engman, T. Sarkissian, H. Aro, M. Wallin, and A.-B. Jonsson, Characterization of motility and piliation in pathogenic Neisseria, BMC Microbiol. 15, 92 (2015).

[22] M. J. Schnitzer, Theory of continuum random walks and application to chemotaxis, Phys. Rev. E 48, 2553 (1993).

[23] A. Verdaguer and J. A. Padro, Velocity cross-correlations and atomic momentum transfer in simple liquids with different potential cores, Phys. Rev. E 62, 532 (2000).

[24] J. R. Blake, A note on the image system for a stokeslet in a no-slip boundary, Math. Proc. Cambridge Philos. Soc. 70, 303 (1971).

[25] L. C. Nitsche, Microhydrodynamics: Principles and selected applications. by Sangtae Kim and seppo J. Karrila, ButterworthHeinemann, Boston, 1991, AlChE J. 40, 739 (1994).

[26] L. Dewenter, T. E. Volkmann, and B. Maier, Oxygen governs gonococcal stability by enhancing the interaction force between type IV pili, Integr. Biol. 7, 1161 (2015).

[27] W. Kob, Computer simulations of supercooled liquids and glasses, J. Phys. Condens. Matter 11, R85 (1999).

[28] G. Volpe and J. Wehr, Effective drifts in dynamical systems with multiplicative noise: a review of recent progress, Rep. Prog. Phys. 79, 053901 (2016).

[29] D. Orsi, A. Fluerasu, A. Moussaid, F. Zontone, L. Cristofolini, and A. Madsen, Dynamics in dense hard-sphere colloidal suspensions, Phys. Rev. E 85, 011402 (2012).

[30] M. A. Charsooghi and A.-R. Moradi, Surface proximity effect in sedimentation investigated by digital holographic microscopy, Appl. Opt. 57, B179 (2018).

[31] S. A. Safran, Statistical Thermodynamics of Surfaces, Interfaces and Membranes (CRC Press, Boca Raton, 2003).

[32] Y. Yifat, N. Sule, and Y. E. A. Lin, Analysis and correction of errors in nanoscale particle tracking using the single-pixel interior filling function (spiff) algorithm, Sci. Rep. 7, 16553 (2017).

[33] R. Zöllner, E. R. Oldewurtel, N. Kouzel, and B. Maier, Phase and antigenic variation govern competition dynamics through positioning in bacterial colonies, Sci. Rep. 7, 12151 (2017). 